\title{
Leadership Training for Oral Health Professionals: A Call to Action
}

\author{
Russell S. Taichman, D.M.D., D.M.Sc.; Joseph W. Parkinson, D.D.S., F.A.G.D.; \\ Bonnie A. Nelson, D.D.S.; Barbara Nordquist, R.D.S.A.; Daphne C. Ferguson-Young, \\ D.D.S., M.S.P.H.; Joseph F. Thompson Jr., M.H.A.
}

Abstract: With dramatic changes in the scope and mode of delivering oral health care on the horizon, a strategic approach to addressing the emerging opportunities and challenges is required. Such an approach will demand new and sustained initiatives to develop leaders with the skills, knowledge, and passion to guide oral health care into the future. The purpose of this position paper is to define the need for leadership training programs for dental and dental hygiene students to become future leaders. Whether these oral health professionals become leaders within a solo or group practice or at the local or national level of their profession, they must be given the mindset and tools to lead. This position paper will describe goals for leadership training and give examples of some programs that currently exist in dental education and other professional settings as the background for a call to action for dental education to provide leadership training opportunities for its students.

Dr. Taichman is Professor, Department of Periodontics and Oral Medicine, Scholars Program in Dental Leadership, University of Michigan School of Dentistry; Dr. Parkinson is Director of Quality Assurance, Department of Restorative Dentistry, University of Missouri-Kansas City School of Dentistry; Dr. Nelson is Assistant Professor and Chair of Pediatric Dentistry, Loma Linda University School of Dentistry, and Department of Hospital Dentistry, Loma Linda University Medical Center; Ms. Nordquist is Vice President, Academic and Professional Relations, KaVo Group; Dr. Ferguson-Young is Associate Professor, Department of Restorative Dentistry, and Director, GPR Program, Meharry Medical College School of Dentistry; and Mr. Thompson is Assistant Dean for Finance and Administration, Medical University of South Carolina College of Dental Medicine. Direct correspondence and requests for reprints to Dr. Russell S. Taichman, Department of Periodontics and Oral Medicine, School of Dentistry, University of Michigan, 1011 North University Ave., Ann Arbor, MI 48109-1078; 734-764-9952 phone; rtaich@umich.edu.

Keywords: dental education, allied dental education, leadership, leadership training

Submitted for publication 2/4/11; accepted 5/25/11

$\mathrm{F}$ orces shaping the oral health professions are leading to changes in the relationships among practitioners, patients, other health care professionals, and related stakeholders. Some of these forces are changing demands for oral health care products and services such as tooth whitening and bonding/veneers; therapeutic options that lead to new therapeutic end points (asking, for instance, whether implants are better treatment options for patients than maintaining periodontally compromised teeth); direct marketing of dental and medical products to consumers; the ability of dental consumers to access and assess health information; changing insurance coverage; electronic health records; the emergence of new oral health care professionals; changing research funding; and increased attention to the need for oral health care for all segments of the U.S. population. Most of these transformations are being driven by influences from outside the dental profession itself: market forces, changed perceptions of what oral and systemic health and well-being mean, technology, research, and discovery. Together, however, they are changing how oral health care providers interact with their patients and their professional peers and should therefore influence how students are being educated. To ensure that the oral health care professions successfully address current and future changes, students must be educated to not only be caring and skilled practitioners but future leaders - an educational approach that can only be achieved by instilling within our institutions a culture that demands and expects leadership.

Oral health care providers have led or participated in many successful policy initiatives. Among these are the implementation of fluoridation programs, a widely celebrated example of public health success. ${ }^{1}$ Similarly, several states have passed legislation mandating oral health exams or assessments upon school entry; in many cases, the efforts behind these requirements were spearheaded by dentists. ${ }^{2}$ 
Other highlights include the development of dental sealant and prevention programs. ${ }^{3}$ Achievement of these and other successes required broad-based support networks among coalitions of stakeholders that provided the leadership, impetus, and environment for change to occur.

Yet while there have been great successes, there have also been challenges. The oral health professions have generally been unable to implement ways to address the basic oral health care needs of a large number of Americans. The reasons are varied and extend beyond oral care to health care in general, yet the inability to provide solutions to the access problem has left many elected officials feeling they have little choice but to consider solutions outside of our profession., ${ }^{4,5}$

A part of the problem lies in the current mission focus of U.S. dental schools. We excel in educating students in the art and science of dentistry by focusing on knowledge in the basic sciences and the development of clinical skills. We also acknowledge those individuals who take on leadership roles within our profession. What we do not do is make a concerted effort to enhance those leadership skills that many students bring to their profession. Nor do we have a systematic approach to nurture the development of leadership skills and a leadership mindset among dental and dental hygiene students that are essential to move the profession forward. The purpose of this position paper is to define the need for leadership training programs for dental and dental hygiene students to become future leaders. Whether these oral health professionals become leaders within a solo or group practice or at the local or national level of their profession, they must be given the mindset and tools to lead. This position paper will describe goals for leadership training and give examples of some programs that currently exist in dental education and in other professional settings as the background for a call to action for dental education to provide leadership training opportunities for its students.

\section{Defining Oral Health Leadership}

Defining the characteristics of leaders has been going on for centuries. Philosophers from Plato on have pondered what distinguishes leaders from others, based on the premise that leadership is rooted in the characteristics possessed by certain individu- als. This trait-based perspective on leadership has dominated much of the study in the field. A number of characteristics have been proposed to distinguish leaders from nonleaders: these include intelligence, dominance, adaptability, persistence, integrity, socioeconomic status, and self-confidence. ${ }^{6}$ An alternative perspective on what constitutes leaders is based upon circumstances: that is, the situation itself molds the leader, so that leaders in one situation may not necessarily be leaders elsewhere.

What constitutes leadership? Noting "there is no infallible step-by-step formula for becoming an effective or transformational leader," Slavkin argued that "leadership signifies the act of making a difference" and that "leadership generally entails sustaining, improving, or changing strategic directions within small or large, simple or complex, organizations." ${ }^{77}$ We would add to this definition that leadership entails the effective communication of a vision (whether shared or not) and an honest and in-depth evaluation of collective experiences and/ or available data so that organizational change may occur in a rational manner.

There are many examples of dentists exhibiting leadership within the scope of their practices, in industry, research, and education, and in professional organizations or governmental bodies. ${ }^{8}$ We might ask what preparation these individuals had for the leadership positions they held. Clearly, reaching a leadership position and exercising leadership in any venue result from many steps an individual has taken as well as circumstances that provided openings. But the process must start with an individual who is receptive to responsibility and willing to take up the mantle of leadership.

\section{Goals for Leadership Training for Oral Health Professionals}

The dramatic transformations in the scope and mode of practice require new and innovative approaches to address these opportunities in how we as a profession do business. Oral health research and discovery are producing new diagnostic and therapeutic options for our patients. Emerging public health issues, how we deliver care and access information, emerging ethical dilemmas, faculty shortages, and changing market forces are likely to test 
the profession's resolve to stay current. But within these trials and challenges there will be opportunities to improve oral health and to improve the stature of the profession by breaking new ground.

Developing leadership skills entails many facets of life. Dental students are well prepared in their study of science, diseases, and current technology, but most dental school curricula lack programs specifically designed to help students learn how to navigate a changing environment or how to successfully manage interpersonal relationships, communication, and decision making skills. Not surprisingly, students enter dental schools with a variety of skills that are considered highly desirable by admissions committees. After graduation, dentists enter practice and are expected to become the leader of the oral health care team. In fact, the Commission on Dental Accreditation's Standard 2-19 reads: "Graduates must understand the basic principles and philosophies of practice management and have the skills to function successfully as the leader of the oral health care team." $"$

At the practice level, if a dentist/leader is unable to positively motivate and share a central vision, the staff's inability to work as a team can cost thousands of dollars each year in lost production and profit. Moreover, the financial loss does not even begin to address the cost that failure of leadership means to patients and to society, which invests heavily in the training of dental professionals and depends on dentists to serve as effective advocates for the oral health needs of our nation. Many case studies have demonstrated that leadership is the missing ingredient in most failing businesses, and dentistry is not alone. ${ }^{10}$ High degrees of staff-related stress and turnover may be the result of a dentist's lack of training in basic leadership and communication skills. A study at the University of Alabama found that dentists who engage in participative decision making processes with staff members create an environment that encourages collaboration, leading to healthy forms of conflict management and increased productivity. ${ }^{11}$ The ability of a dentist to be an effective leader who can recruit and retain excellent employees creates an environment that carries over to high-quality patient care and services. Satisfied patients also look to their dentists to serve as role models and mentors.

Dentists also have opportunities to serve as leaders or change agents within their communities and neighborhoods. Numerous opportunities exist in which oral health professionals can lend their knowledge and support to local efforts that improve health and well-being. For example, water fluoridation and sealant programs are often of great benefit to communities as dental caries remains one of the most prevalent health care issues. Dentists serving at the local level may also function in important capacities by helping to improve the overall health of the population as evidence mounts indicating a possible link between poor oral health and other systemic conditions. ${ }^{12-15}$ In these two areas, dentists may serve large population segments that are underserved, particularly in urban and rural areas, by medical and dental/oral health professionals. Dentists can help by participating in oral health care education and outreach programs.

Within dental school/oral health curricula, training future leaders of the profession has generally been relegated to the training of academic and scientific leaders. A long history of programs, events, and support mechanisms are available to guide/mentor aspiring oral health leaders into the critical roles of academic dentistry. While much has been written to address faculty shortages, at least there are established venues and role models that can facilitate the recruitment and retention of these valuable members of our profession in these roles. Yet beyond these venues and targeted leadership positions there are few opportunities.

Table 1 presents our vision of some characteristics of an ideal leadership training program for oral health care professionals. Leadership training programs specifically designed to foster studentfaculty dialogue focused on leadership development are starting to emerge. In many ways, these programs provide informal settings for collegial discussions that would not normally occur within a classroom or clinical setting. In this respect, it is envisioned that many of the institutional barriers to learning can be

\section{Table 1. Characteristics of an ideal training program in leadership for oral health professionals}

- Focuses development on areas of strength and interest rather than building competence in weak areas exclusively.

- Focuses on active adult learning.

- Reflects institution's long-term commitment to build leadership capacity in alumni.

- Serves as an opportunity to bring together resources and individuals who have not traditionally participated in oral health education (e.g., business leaders outside of dentistry, motivational speakers, journalists). 
overcome. Moreover, focus on leadership as a venue for scholarship at both local and national levels is possible and may ultimately provide a vehicle for addressing faculty shortages. ${ }^{16,17}$ Since most dental school applicants are not specifically targeted and recruited into the profession for their desire to become faculty members or leaders, enhanced exposure to faculty and adjunctive faculty members in leadership roles is bound to create a climate that sends a very different message to students. We believe that this exposure may stimulate participants to seek career paths they may not have otherwise considered.

\section{Leadership Training in Other Professional Schools}

As in dentistry, leadership training in medical education would be desirable in many facets of physicians' career as they interact with patients and the many facets of the health care industry. In the article "Leadership: 'They Never Taught Me This in Medical School," Fruge et al. outline a leadership curriculum for fellows at the Texas Children's Hospital. ${ }^{18}$ The approach presented is case-based in seminar style. O'Connell and Pascoe evaluated eight schools incorporating medical education for the twenty-first century content: leadership and teamwork. They believed that leadership training worked well if incorporated into clinical activities where the skills could be used..$^{19} \mathrm{~A}$ learning community is another way to integrate leadership into medical education; when the students get involved in this system, learning is done by both the students and other members of the learning community including faculty members and other stakeholders. ${ }^{20,21}$ The most common stated goals of these organizations are to improve communication and teamwork, establish academic support networks, and promote leadership development.

Yet other models exist. As in the education of other professionals, there are many programs that students can be involved in to learn better leadership skills $^{22,23}$ and many articles that address the needs and programs for medical education and medical resident education. $^{24,25}$

Like medicine and dentistry, as business school graduates enter the workforce, leadership training in the corporate world is often tailored to suit niche needs. These needs often represent the distinctive management and leadership approach of the organization. One large organization with a highly evolved leadership program regularly updates this program to remain current and relevant. As with most corporations that have professional development programs, most include some elements relating to promoting and nurturing leaders. Often companies, especially smaller companies, use outside-developed, more generic programs that were not necessarily tailored to represent the culture and ethos of the specific company.

Business schools are often seen as leaders in general management skills and in leadership. Bennis's pioneering work laid the groundwork for many of the modern reforms in business education. ${ }^{26} \mathrm{His}$ approach includes recognition of the role of judgment and emotion in decision making and the importance of ethics. Still, many business schools today have been criticized for not teaching effective leadership, so a new approach is required focusing research on phenomena and problems of enduring importance and building curricula that are evaluated, in part, by how well they actually prepare students to be effective in practicing the profession.

Increasingly, corporations are developing their own future generations of leaders through internally developed educational programs. These programs seek to capitalize on their own successful leadership and results in order to drive these skills further down the management team. Many of these programs have a structured curriculum covering the many management disciplines, taught by managers from across the corporation. These programs often rely on reviewing and learning from the successes of the company in order to teach desired actions and behavior. Thus, the next generations of leaders are nurtured within the framework of a successful corporation.

\section{Examples of Leadership Training for Dental and Dental Hygiene Students}

What should leadership training in dentistry be in the context of a need for oral health leaders? Is the best practice for dentistry to look to business schools or other professional programs for leadership content? Is management training leadership training? Should the focus be on leading a dental practice or training dentists/dental hygienists to be leaders at local, state, and national levels? Is a research experience leadership training in and of itself? We assert that the need is much greater and goes beyond 
existing practices. Dental schools must develop their own curricula.

As leadership training is becoming more prevalent in other industries, there is vast divergence in the perceived need or scope. As such, the state of the art for dentistry developed specifically to address the needs of dentists is still in its infancy. How have leadership training programs been incorporated and conceived within dental school communities? Victoroff et al. found that dental students overwhelmingly view leadership as a part of their profession and would be willing to participate in leadership training programs if they existed within their institutions. ${ }^{8,27}$ Interestingly, in 2000 nearly 70 percent of responding dental school deans reported that leadership training was the most crucial aspect in preparing to become a dean. ${ }^{28} \mathrm{In}$ fact, this area of study and experience was perceived by the respondents to be more important than the ability to teach dentistry, knowledge of budget and finance, and conducting research.

The concept that leadership instruction in dental schools needs to be developed for dentistry is a critical departure from how many industries provide leadership training. An eleven-week program was recently reported at the School of Dentistry at the University of Southern California. ${ }^{29}$ This program combines much of the traditional industry-wide aspects of leadership training including communication styles and listening, people management, creating and leading a team, building coalitions, conflict management, and fiscal management. Kalenderian et al. reported on the recent development of a third-year course titled "Dental Health Care Delivery: Concepts of Oral Health Leadership" at Harvard School of Dental Medicine. ${ }^{30}$ The course focused on management and leadership concepts. The management topics focused on practice management, patient-centered care, public health dentistry, and systems analysis of medical errors, including how to improve patient safety. The leadership topics included ethics, systemic intervention to reduce oral health disparities, cultural awareness, building a team environment, interpersonal communication skills, and conflict management. The course met weekly for a total of fifty-four hours over twenty-two weeks, with the time split evenly between management and leadership.

At the University of Michigan, a Scholars Program in Dental Leadership (SPDL) has been developed that encompasses all four years of the dental curriculum to provide sufficient time for group learning and the development of capstone projects targeting the interests of the participants themselves. ${ }^{31}$ The SPDL was designed to wrap around the curriculum so that program events would occur mainly after hours. Program enrollment is based upon an application process that requires applicants to seek out partnering mentors. Program activities include yearly kick-off events, student-held organizational and progress meetings focused on project/capstone development, and monthly leadership training events in which guest speakers are asked to participate. Currently, this after-school program is serving as part of a curriculum design launched in fall 2010 in which all dental students have time designated across all four years for one of three pathways: health care delivery, research, or leadership. Students in the leadership pathway focus on critical issues for the profession, participate in a leadership journal club, and develop projects that hone their leadership skills in addition to content provided in the SPDL program.

Several questions must be answered in order for schools to implement leadership training programs. First, do students value the development of leadership skills, and will they participate? Victoroff et al. found that dental students view leadership as a part of their profession and would participate in leadership training programs within their institutions. ${ }^{8}$ Second, how would such a program fit within an already packed curriculum? That is, should leadership training be a distinct course, or should content be embedded within existing courses or as an extracurricular activity? Third, what would this curricular supplement cost, and how would those expenses be managed? Finally, who will provide the content? Are existing faculty members capable of providing leadership training, or would it be best to partner with other programs? These aspects of program design are discussed in an accompanying article. ${ }^{32}$

\section{Conclusions}

Stand-alone leadership training for dental and allied dental students is a new educational opportunity that is currently unexplored in most institutions, yet many aspects of the current curriculum could be used or expanded upon as a basis for leadership training. These include practice management, diversity education, and research training.

We believe that a focus on leadership studies would change the culture of our profession in a positive direction. Stating that we as a profession value 
leadership and providing opportunities for students to exercise new skills would enrich education for students and faculty members alike. By focusing on challenging group or individual projects, we believe that new alliances from across campuses and professions will be forged. The benefits of these cannot be completely envisioned at this point. It would be a mistake for most of us to look to other professionals, be it business or medicine, to provide content. However, our culture and our students will benefit from these experiments with new and exciting educational opportunities. The important point is to start a discussion focused on leadership. Different individuals and schools will have different definitions, outcome measures, and methods to achieve leadership competencies, yet it is through discussion that we will change our profession.

\section{Acknowledgments}

The authors would like to thank their parent institutions and deans for support during their participation in the American Dental Education Association (ADEA) Leadership Institute. The authors would like to acknowledge the help of Dr. Cecile Feldman, dean, University of Medicine \& Dentistry/New Jersey Dental School; Dr. Karl Haden, president, Academy for Academic Leadership, and his staff; and other fellows in the ADEA Leadership Institute. Dr. Taichman is supported by grants from the National Institutes of Health (DK082481, RC1DE020721, AR056893, CA093900) and the Department of Defense (PC073952). Sincere appreciation also goes to ADEA and Alpha Omega Foundation for supporting Dr. Taichman as a Leonard Abrams Scholar during his participation in the ADEA Leadership Institute. Finally, we would like to acknowledge the support and encouragement of our families and our students, for whom this work is intended.

\section{REFERENCES}

1. Easley MW. Celebrating 50 years of fluoridation: a public health success story. Br Dent J 1995;178:72-5.

2. Policy on mandatory school-entrance oral health examinations. Pediatr Dent 2008;30:26-7.

3. Buonocore MG. Caries prevention in pits and fissures sealed with an adhesive resin polymerized by ultraviolet light: a two-year study of a single adhesive application. J Am Dent Assoc 1971;82:1090-3.

4. Hathaway KL. An introduction to oral health care reform. Dent Clin North Am 2009;53:561-72.

5. Harris T. The U.S. oral health workforce in the coming decade: workshop summary. Washington, DC: Institute of Medicine, 2009.
6. Bass B, Bass R. The Bass handbook of leadership: theory, research, and managerial applications. 4th ed. New York: Free Press, 2008.

7. Slavkin H. Leadership for health care in the 21 st century: a personal perspective. J Healthcare Leadership 2010;2:35-41.

8. Victoroff KZ, Schneider K, Perry C. Leadership development for dental students: what do students think? J Dent Educ 2008;72(9):982-8.

9. Commission on Dental Accreditation. Accreditation standards for dental education programs. Chicago: American Dental Association, 2007.

10. Schumann T. Leadership: the young dentist's secret to success. J Mich Dent Assoc 2002;84:22.

11. Chilcutt AS. Exploring leadership and team communication within the organizational environment of a dental practice. J Am Dent Assoc 2009;140:1252-8.

12. Hollister MC, Weintraub JA. The association of oral status with systemic health, quality of life, and economic productivity. J Dent Educ 1993;57(12):901-12.

13. Beck JD, Offenbacher S. Oral health and systemic disease: periodontitis and cardiovascular disease. J Dent Educ 1998;62(10):859-70.

14. Amar S, Han X. The impact of periodontal infection on systemic diseases. Med Sci Monit 2003;9:291-9.

15. Iacopino AM. Translation of oral-systemic science to practice. J Can Dent Assoc 2008;74:496-7.

16. Haden NK, Beemsterboer PL, Weaver RG, Valachovic RW. Dental school faculty shortages increase: an update on future dental school faculty. J Dent Educ 2000;64(9):657-73.

17. Nunn PJ, Gadbury-Amyot CC, Battrell A, Bruce SI, Hanlon LL, Kaiser C, Purifoy-Seldon B. The current status of allied dental faculty: a survey report. J Dent Educ 2004;68(3):329-44.

18. Fruge E, Mahoney DH, Poplack DG, Horowitz ME. Leadership: "they never taught me this in medical school." J Pediatr Hematol Oncol 2010;32:304-8.

19. O’Connell MT, Pascoe JM. Undergraduate medical education for the 21st century: leadership and teamwork. Fam Med 2004;36(Suppl):S51-S56.

20. Bicket M, Misra S, Wright SM, Shochet R. Medical student engagement and leadership within a new learning community. BMC Med Educ 2010;10:20.

21. Ferguson KJ, Wolter EM, Yarbrough DB, Carline JD, Krupat E. Defining and describing medical learning communities: results of a national survey. Acad Med 2009;84:1549-56.

22. The MD/MBA program at Dartmouth. At: http://dms. dartmouth.edu/mdmba/faqs/. Accessed: August 12, 2010.

23. University of Queensland, Australia. Medical leadership program. At: www2.som.uq.edu.au/SOM/CURRENTSTUDENTS/STULEADERSHIP/Pages/default.aspx. Accessed: August 12, 2010.

24. Bligh J, Brice J. Leadership in medical education. BMJ 2010;340:2351.

25. Hemmer PR, Karon BS, Hernandez JS, Cuthbert C, Fidler ME, Tazelaar HD. Leadership and management training for residents and fellows: a curriculum for future medical directors. Arch Pathol Lab Med 2007;131:610-4. 
26. Bennis W. On becoming a leader. New York: AddisonWesley, 2010.

27. Victoroff KZ, Schneider K, Perry C. Tomorrow's leaders, starting today: a pilot leadership development program for dental students. J Dent Educ 2009;73(3):311-8.

28. Brundo GC. Preparing to be a dean. J Dent Educ 2000;64(6): 430-2.

29. Slavkin HC, Lawrence L. Incorporating leadership knowledge and skills into the dental education community. J Dent Educ 2007;71(6):708-12.
30. Kalenderian E, Skoulas A, Timothé P, Friedland B. Integrating leadership into a practice management curriculum for dental students. J Dent Educ 2010;74(5):464-71.

31. Taichman RS, Green TG, Polverini PJ. Creation of a scholars program in dental leadership (SPDL) for dental and dental hygiene students. J Dent Educ 2009;73(10): 1139-43.

32. Taichman RS, Parkinson JW, Nelson BA, Nordquist B, Ferguson-Young DC, Thompson JF Jr. Program design considerations for leadership training for dental and dental hygiene students. J Dent Educ 2012;76(2):192-9. 